\title{
Mejora de la tenacidad de un acero de ultraalto contenido en carbono mediante unión por laminación con un acero al cromo y molibdeno
}

\author{
M. Pozuelo*, F. Carreño* y O.A. Ruano*
}

\begin{abstract}
Resumen Se procesaron mediante unión por laminación, dos materiales compuestos laminados de 10 capas: cinco capas de un acero de ultraalto contenido en carbono con 1,35\% C, alternadas con otras cinco de un acero al cromo y molibdeno. La laminación en caliente consolida la unión entre las capas y afina la microestructura, reduciendo el tamaño de grano y eliminando las zonas de perlita presentes inicialmente. Se realizaron ensayos de impacto Charpy para evaluar su resistencia al impacto. El material compuesto laminado mejoró sustancialmente la tenacidad del acero de ultraalto carbono, además de duplicar la resiliencia del acero al cromo y molibdeno en la orientación "en serie". Estos resultados ponen de manifiesto la importancia de las intercaras en las propiedades mecánicas de los materiales compuestos.
\end{abstract}

Palabras clave Acero de ultraalto carbono. Materiales compuestos laminados. Laminación en caliente. Tenacidad. Impacto.

\section{Toughness improvement of an ultrahigh carbon steel by rolling bonding with a $\mathrm{Cr}-\mathrm{Mo}$ steel}

\begin{abstract}
Laminated composite materials containing five layers of ultrahigh carbon steel, $1.35 \% \mathrm{C}$, and five layers of a Cr-Mo steel were processed by roll bonding. The rolling procedure improves the bonding of layers and refines the microstructure, diminishing grain size and removing the pearlitic zones. Charpy impact tests were carried out to evaluate the impact properties. Toughness of the laminated composite was highly improved respect to the ultrahigh carbon steel. Moreover, it was double than that of the $\mathrm{Cr}$-Mo steel in the arrester orientation. These results reveal the important role of the interfaces in the mechanical properties of the composite materials.
\end{abstract}

Keywords Ultrahigh carbon steel. Laminated composite materials. Hot rolling Toughness. Impact.

\section{INTRODUCCIÓN}

La baja tenacidad a fractura de los aceros de ultraalto contenido en carbono (AUAC), de composición hipereutectoide (conteniendo 0,8-2,1\% C) ha sido un condicionante clave para su uso estructural. La obtención de valores de tenacidad elevados a temperatura ambiente ha sido objeto de numerosos estudios ${ }^{[1-4]}$.

La unión de aceros resistentes con otros materiales más dúctiles, aceros suaves y al cromo y molibdeno (ACM), formando compuestos laminados de acero, ha permitido obtener resultados de resistencia al impacto y a la fractura muy relevantes ${ }^{[5-8]}$.

En este trabajo, se presenta el estudio de un material compuesto laminado de aceros AUAC y
ACM que ha sido conformado mediante unión por laminación en caliente. Se ha prestado especial atención al estado microestructural y mecánico de las intercaras para que favorezca la delaminación, lo cual será determinante para conseguir buenas propiedades mecánicas.

\section{MATERIALES Y PROCEDIMIENTO EXPERIMEN- TAL}

Se han procesado mediante laminación en caliente dos tochos, denominados "DG10x" y "DG10y", y constituidos por cinco capas de un acero de ultraalto contenido en carbono AUAC, "D" (1,35 \% $\mathrm{C})$, alternadas con cinco capas de un acero al cromo y molibdeno ACM, "G".

(*) Departamento de Metalurgia Física, CENIM, CSIC, Av. Gregorio del Amo 8, 28040 Madrid, España. 
La composición, en \% en masa, de los aceros constituyentes es la siguiente: 1,35 \% C-0,54 \% Cr $-0,25 \% \mathrm{Mn}-0,17 \% \mathrm{Si}$ para el acero D y $0,15 \% \quad \mathrm{C}-1,05 \quad \% \quad \mathrm{Cr}-0,4 \quad \% \quad \mathrm{Mn}-0,18 \quad \%$ Mo- $0,031 \% \mathrm{Ni}-0,23 \% \mathrm{Si}$ para el acero G. Ambos aceros fueron laminados en forma de planchas de espesores de 4 y $2 \mathrm{~mm}$ para el AUAC y el ACM, respectivamente. Posteriormente, se apilaron de forma alternada hasta un espesor total de $28,5 \mathrm{~mm}$.

Los dos compuestos fueron sometidos a un proceso de laminación en caliente. El "DG10x" se mantuvo $30 \mathrm{~min}$ a $775^{\circ} \mathrm{C}$ y se laminó en cuatro series de 6, 4, 4 y 3 pasadas, con una reducción aproximada de $1 \mathrm{~mm}$ por pasada hasta un espesor final de 11,5 mm. El tiempo de calentamiento en el horno entre cada ciclo fue de aproximadamente 10 min. El "DG10y" se laminó a $770^{\circ} \mathrm{C}$ en cinco series de 5, 4, 4, 2 y 3 pasadas, con una reducción aproximada de $1 \mathrm{~mm}$ en todas las pasadas salvo en las de la última serie que fue de $0,5 \mathrm{~mm}$. El tiempo de calentamiento en el horno fue de aproximadamente 10 min entre cada ciclo. Como resultado final se obtuvo un lingote de $11,5 \mathrm{~mm}$ espesor, que quedó ligeramente curvado y fue posteriormente enderezado en el macho pilón a temperatura ambiente. La deformación real acumulada en el procesado de ambos compuestos fue de $\varepsilon=-0,9$.

Las muestras seleccionadas se cortaron, pulieron y atacaron con Nital al $2 \%$ para revelar la microestructura y observarse en el microscopio electrónico de barrido (MEB), donde se realizaron también microanálisis de las intercaras de ambos compuestos. Adicionalmente, se realizaron ensayos de microdureza Vickers bajo carga de $100 \mathrm{~g}$.

Se mecanizaron probetas tipo Charpy con entalla en $\mathrm{V}$ de $2 \mathrm{~mm}$, tanto de los materiales constituyentes $\left(10 \times 5,4 \times 55 \mathrm{~mm}^{3}\right.$ para el $\mathrm{D}$ y de $10 \times 3,5$ $\times 55 \mathrm{~mm}^{3}$ para el G) como de los dos compuestos laminados $\left(10 \times 10 \times 55 \mathrm{~mm}^{3}\right)$, tanto en la orientación "en serie" como "en paralelo". Se ensayaron a impacto con un péndulo de $294 \mathrm{~J}$.

\section{RESULTADOS Y DISCUSIÓN}

\subsection{Microestructura}

En la figura 1 se puede observar la microestructura inicial de los aceros constituyentes, antes de formar parte de los materiales compuestos laminados. El AUAC "D" (Fig. 1a) presenta una microestructura globular, con carburos de hierro redondeados, aunque también se observan algunos con formas más alargadas. El tamaño de grano ferrítico es aproximadamente de $10 \mu \mathrm{m}$ con carburos proeutectoides en sus bordes y otros de menor tamaño en su interior. El ACM, "G", (Fig. 1b) presenta un tamaño de grano ferrítico de aproximadamente 10 $\mu \mathrm{m}$ y la presencia de algunas zonas perlíticas.

En la figura 2 la microestructura de estos aceros ha variado como consecuencia del procesado. Los cambios experimentados son muy similares en los dos compuestos. El tamaño de grano ferrítico en el AUAC, "D", ha disminuido, siendo ahora inferior a $5 \mu \mathrm{m}$ y los carburos son también de menor tamaño, inferiores a $1 \mu \mathrm{m}$. En el ACM, "G", el tamaño de grano ferrítico también se ha afinado, presentando formas más alargadas según la dirección de laminación. La mayoría de las zonas perlíticas han desaparecido, y los carburos están globulizados y son inferiores a $1 \mu \mathrm{m}$. En las figuras $2 \mathrm{a}$ y $2 \mathrm{~b}$ se muestran, respectivamente, una intercara de las más exteriores y una de las más interiores del laminado "DG10x". La intercara exterior presenta
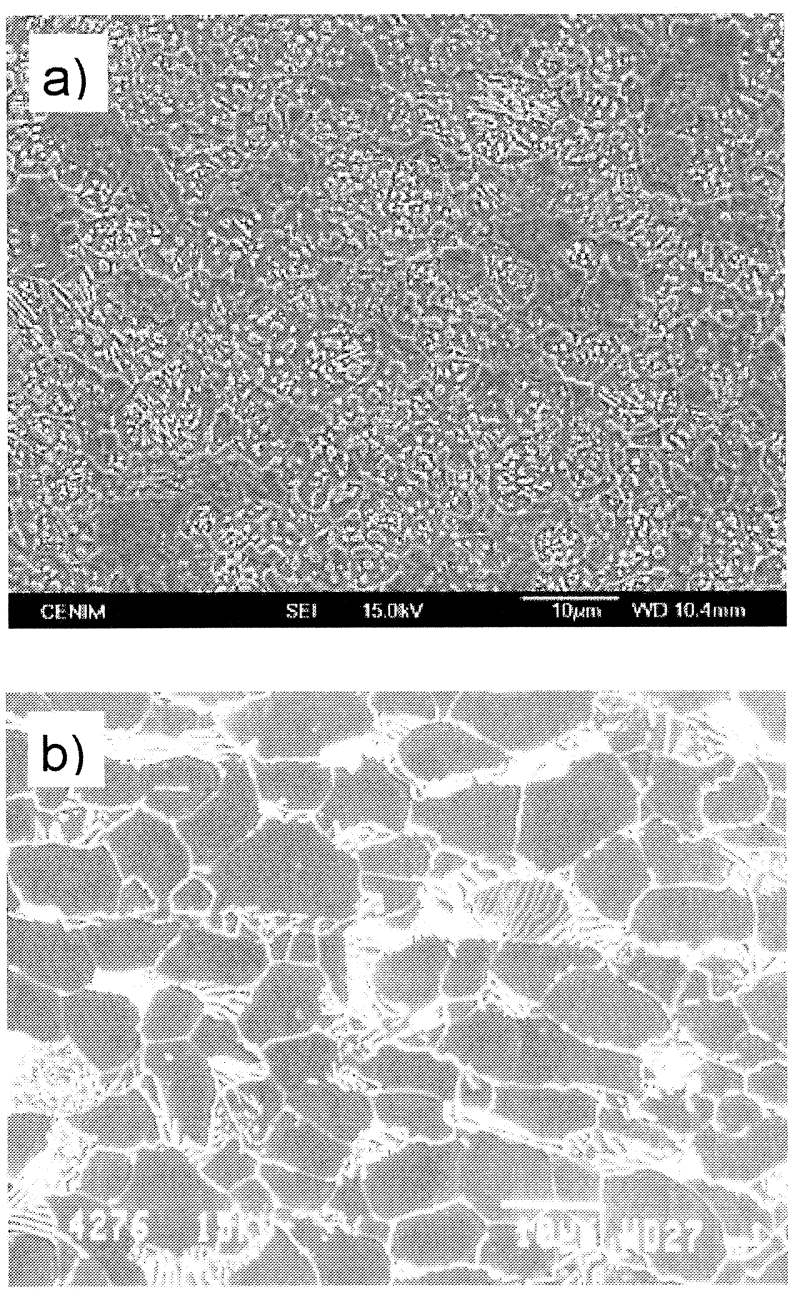

Figura 1. Microestructuras iniciales observadas en el MEB: a) AUAC, D y b) ACM, G.

Figure 1. Initial microstructures observed in the SEM: a) UHCS, D and b) CMS, G.

Rev. Metal. Madrid Vol. Extr. (2005) 107-110 
Mejora de la tenacidad de un acero de ultraalto contenido en carbono mediante unión por laminación con un acero al cromo y molibdeno M. Pozuelo, F. Carreño y O.A. Ruano

una banda de $3 \mu \mathrm{m}$ de espesor, con la presencia de algún poro y sin difusión de carbono entre las capas. En la intercara interior (Fig. 2b), se puede observar una mayor proporción de porosidad.

En la figura 2c se muestra una intercara del material "DG10y"; en este caso no se encontró ninguna diferencia entre intercaras interiores y las más exteriores del laminado. Se observa una intercara fina y limpia, sin presencia de poros. En la figura $2 \mathrm{~d}$ se muestra el microanálisis comparativo entre dos intercaras de ambos laminados. Se observa la presencia de un pico de oxígeno en la intercara del "DG10x", que no aparece en la del "DG10y". Esto indica la presencia de óxidos en la intercara del "DG10x".

\subsection{Ensayos de impacto}

La tabla I muestra los resultados de los ensayos Charpy realizados en los materiales laminados en
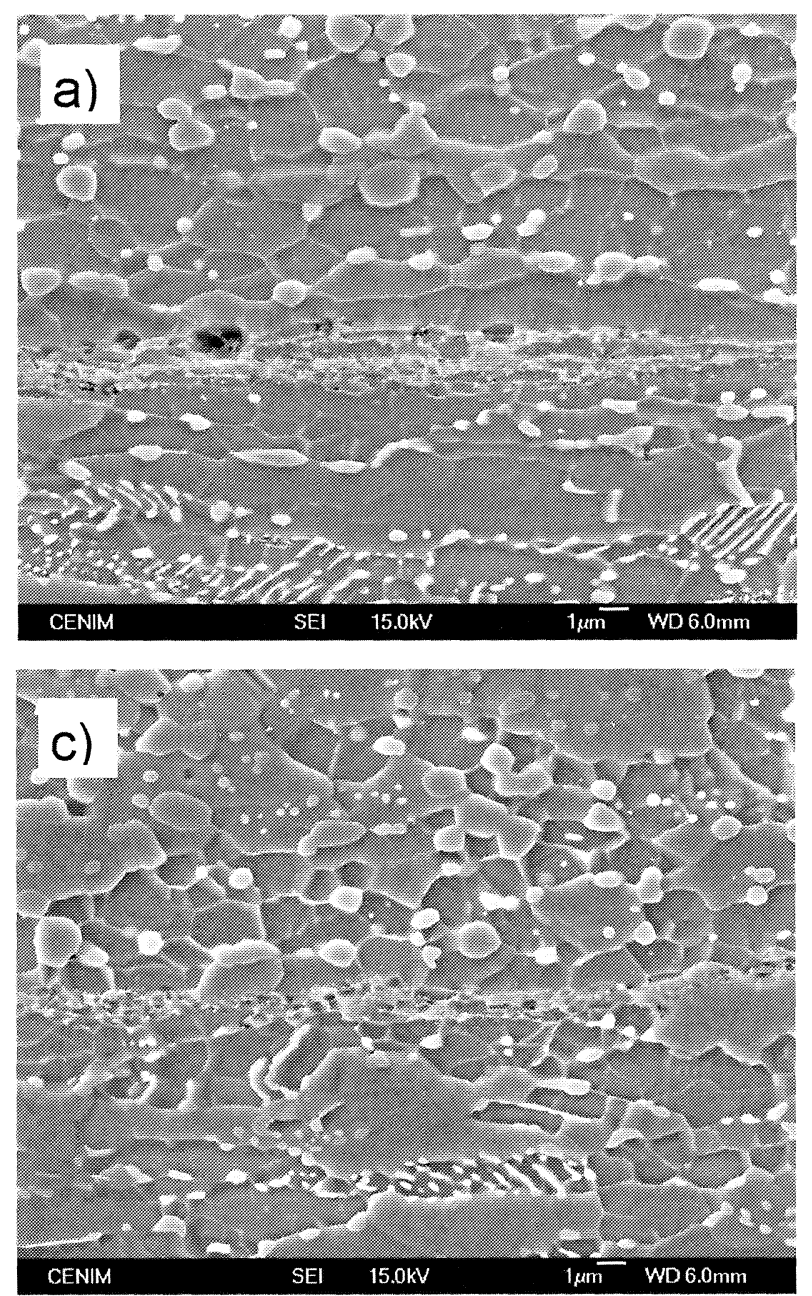

Tabla I. Resiliencia de los materiales compuestos laminados "DG10x" y "DG10y".

Table I. Resilience of the laminated composite materials "DG10x" and "DG10y"

\begin{tabular}{lccr}
\hline Material & \multicolumn{3}{c}{ Resiliencia $\left(\mathrm{kJ} / \mathrm{m}^{2}\right)$} \\
\cline { 2 - 4 } & Probeta 1 & Probeta 2 & Media \\
\hline DG10x "en serie" & 1.901 & 2.097 & 1.999 \\
DG10x "en paralelo" & 245 & 245 & 245 \\
DG10y "en serie" & 1.950 & 1.925 & 1.937 \\
DG10y "en paralelo" & 258 & 245 & 251 \\
\hline
\end{tabular}

la orientación "en serie" y "en paralelo". Los materiales laminados en ambas orientaciones mejoran sustancialmente la tenacidad del AUAC, "D" $\left(<100 \mathrm{~kJ} / \mathrm{m}^{2}\right)$. Además, los valores obtenidos para ambos laminados en la orientación más favorable, "en serie", superan también el obtenido para el
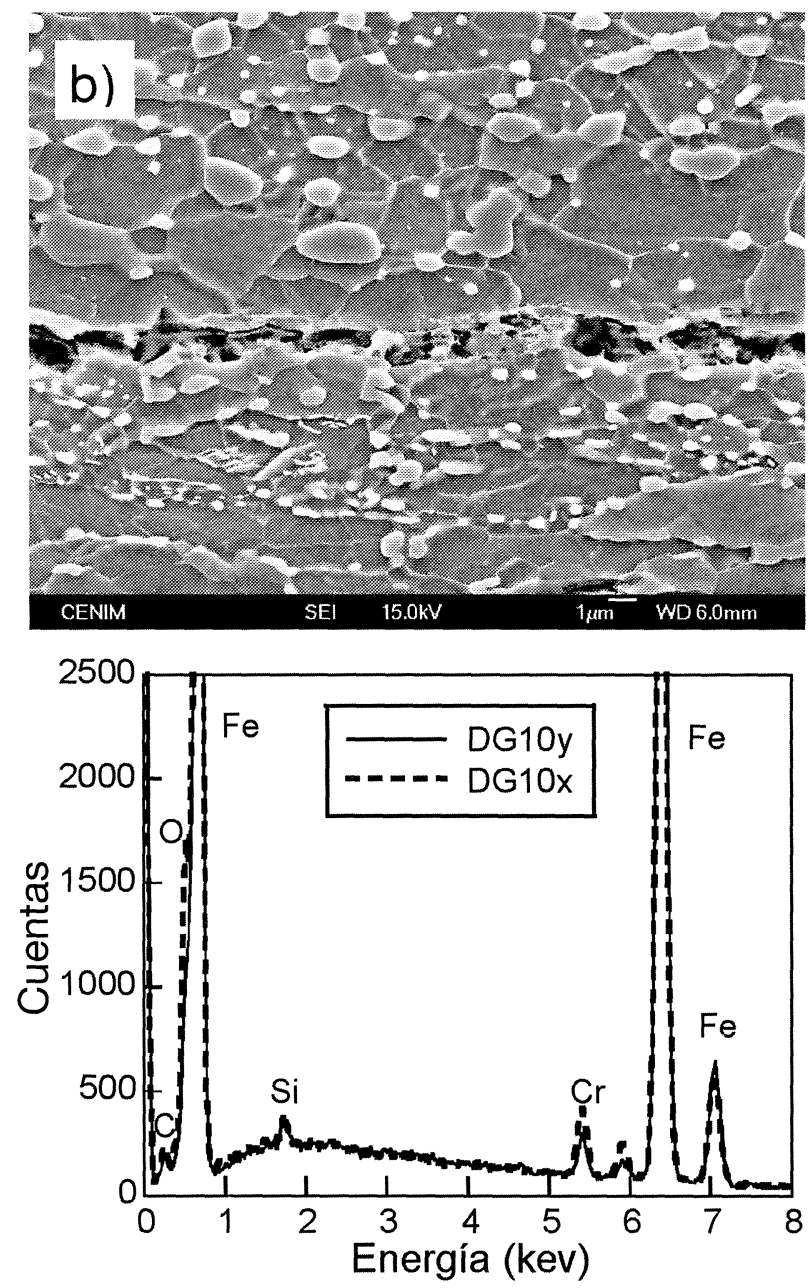

Figura 2. a) Intercara exterior del laminado "DG10x", b) intercara interior del laminado "DG10x", c) intercara del laminado "DG10y" y d) microanálisis comparativo entre dos intercaras de ambos laminados.

Figure 2. a) Exterior interface of the laminate "DG10x", b) interior interface of the laminate "DG10x" c) interface of the laminate "DG10y" and d) comparative microanalysis between two interfaces of both laminates. 
Mejora de la tenacidad de un acero de ultraalto contenido en carbono mediante unión por laminación con un acero al cromo y molibdeno M. Pozuelo, F. CARreño y O.A. Ruano

ACM, "G" $\left(1.190 \mathrm{~kJ} / \mathrm{m}^{2}\right)$. Se puede observar, también, una ligera dispersión entre los valores de las dos probetas del laminado "DG10x" en la orientación "en serie".

Si se comparan los dos materiales laminados en la orientación más favorable, "en serie", el laminado "DG10x" es un poco más resistente al impacto que el "DG10y". La razón de que, en este último, el valor de energía absorbida sea menor se puede deber a dos contribuciones. Por un lado, este material fue enderezado en el macho pilón para eliminar parte de la curvatura resultante en su procesado, lo que pudo aumentar su acritud y, por tanto, disminuir su tenacidad a fractura. Para comprobarlo, se realizaron ensayos de dureza Vickers sobre el material más frágil, en este caso el AUAC, que es el que induce fragilidad al resto del material compuesto. Los resultados obtenidos evidencian el hecho de una mayor acritud en el laminado "DG10y", ya que la dureza del AUAC de este laminado, $255 \mathrm{HV}$, es superior a la del AUAC del laminado "DG10x", 235 HV. Por otro lado, el estado de sus intercaras también fue diferente. La baja densidad de porosidad en las intercaras del "DG10y" refleja un estado de la unión fuerte. En este caso, el efecto que introducen las intercaras en la desviación de la grieta es algo menor. Las intercaras del laminado "DG10x", por la elevada presencia de poros, reflejan una peor unión, lo que se traduciría en una mayor resistencia a la propagación de la grieta ya que ésta puede desviarse más fácilmente por mecanismos de delaminación entre las capas. Además, la precipitación de óxidos sobre estas intercaras, induce fragilidad a las mismas, lo que favorece aún más la delaminación, desviando la grieta e inhibiendo la propagación de la misma a través de todo el espesor de la probeta ${ }^{[8]}$. Esta delaminación es la causante del aumento de tenacidad de estos materiales.

\section{CONCLUSIONES}

Se han procesado mediante laminación en caliente dos materiales compuestos laminados, AUAC alternado con ACM. Ambos materiales laminados, con una microestructura más fina y globular como consecuencia del procesado, han mejorado sustancialmente las propiedades de impacto del AUAC e incluso la del acero más dúctil, ACM, en la orientación "en serie". De hecho, el valor de energía Charpy absorbida de los materiales compuestos laminados prácticamente duplica el valor del ACM, ya de por sí muy tenaz. Las ligeras variaciones de energía Charpy absorbida entre las diferentes probetas se atribuyen a diferencias en la unión de las intercaras. Esta unión favorece mecanismos extrínsecos de fractura como delaminaciones entre capas. Estas delaminaciones, responsables del aumento de tenacidad, desvían la propagación de la grieta, evitando así la rotura catastrófica del material.

\section{Agradecimientos}

Los autores desean expresar su agradecimiento al Dr. Manuel Carsí por el apoyo prestado a través del proyecto CICYT MAT2000-2017-C02-01. También se quiere agradecer a D. Luis del Real Alarcón, por la soldadura de los tochos antes de ser laminados, a D. Fernando Federico González por su colaboración en la laminación en caliente y a D. J. Chao por su asistencia durante los ensayos Charpy.

\section{REFERENCIAS}

[1] O.D. Sherby, T. Oyama, D.W. Kum, B. Walser y J. WADSWORTH, J. Met. 37 (1985) 50-56.

[2] D.R. Lesuer, C.K. Syn y O.D. ShERby, Acta Metall. Mater. 43 (1995) 3827-3835.

[3] A. Fernández-Vicente, M. Carsí, F. Peñalba, E. TalefF y O.A. Ruano, Mater. Sci. Eng. A 335 (2002) 175-185.

[4] M. Pozuelo, F. Carreño y O.A. Ruano. Vill Congreso Nacional de Propiedades Mecánicas de Sólidos, Gandía, Valencia, Junio, 2002, Resúmenes, pp. 943-953.

[5] F. Carreño, M. Pozuelo, J. Chao y O.A. Ruano. Rev. Metal. Madrid 37 (2001) 130-134.

[6] F. Carreño, J. Chao, M. Pozuelo y O.A. Ruano, Scripta Mater. 48 (2003) 1135-1140.

[7] M. Pozuelo, F. Carreño y O.A. Ruano, Bol. Soc. Esp. Cerám. Vidrio 43 (2004) 207-210.

[8] M. Pozuelo, F. Carreño y O.A. Ruano, Mater. Sci. Forum 426-432 (2003) 883-888. 